\title{
A Catecholamine-Secreting Skull Base Sinonasal Paraganglioma Presenting with Labile Hypertension in a Patient with Previously Undiagnosed Genetic Mutation
}

\author{
Samuel Hahn, M.D. ${ }^{1}$ James N. Palmer, M.D. ${ }^{1}$ Nithin D. Adappa, M.D. ${ }^{1}$ \\ ${ }^{1}$ Department of Otorhinolaryngology-Head and Neck Surgery, \\ Hospital of the University of Pennsylvania, Philadelphia, Pennsylvania \\ J Neurol Surg Rep 2012;73:19-24.

\begin{abstract}
Address for correspondence and reprint requests Samuel Hahn, M.D., Department of Otorhinolaryngology-Head and Neck Surgery, Hospital of the University of Pennsylvania, 3400 Spruce Street, 5 Silverstein, Philadelphia, PA 19104 (e-mail: samuel.hahn@uphs.upenn.edu).
\end{abstract}

\begin{abstract}
Keywords

- head and neck paraganglioma

- sinonasal paraganglioma

- endoscopic skull base resection

- succinate dehydrogenase mutation
\end{abstract}

Paragangliomas of the head and neck are uncommon tumors arising from specialized neural crest cells associated with autonomic ganglia. They account for only $0.6 \%$ of all tumors within the head and neck region with functioning paragangliomas seen in only 1 to $3 \%$ of those cases., ${ }^{1,2}$ The distribution of paragangliomas tends to parallel the course of the autonomic system. They can be categorized into two types based on their functionality: catecholamine-secreting chromaffin tumors and the nonfunctional nonchromaffin tumors. The majority of head and neck paragangliomas belongs to the latter group. Carotid body tumors are the most common paraganglioma of the head and neck region followed by jugulotympanic and vagal paragangliomas. Head and neck paragangliomas have also been reported in the larynx, trachea, orbit, and sinonasal cavities. ${ }^{3}$ Succinate dehydrogenase (SDH) complex (A to D) mutations have been associated with increased risk of tumors including paragangliomas and pheochromocytomas. Tumors associated with the SDHB mutations tend to be extra-adrenal and can occur in the head and neck region. ${ }^{4}$

There have been approximately only 35 reported cases of paragangliomas originating from the sinonasal region. ${ }^{5}$ Pathologic studies have shown paraganglionic tissue to be present in the pterygopalatine fossa as well as the close anatomic relation with the pterygoid ganglion. ${ }^{6}$ There have been only three reported cases of functional sinonasal paragangliomas since $1957 .^{7-9}$ We present a case of a rare functioning sinonasal paraganglioma of the skull base presenting with labile hypertension. received

September 23, 2011

accepted

November 23, 2011

published online

February 17, 2012
Copyright $\odot 2012$ by Thieme Medical Publishers, Inc., 333 Seventh Avenue, New York, NY 10001, USA.

Tel: +1(212) 584-4662.
DOI http://dx.doi.org/ 10.1055/s-0032-1301408. ISSN 2193-6358. 
A
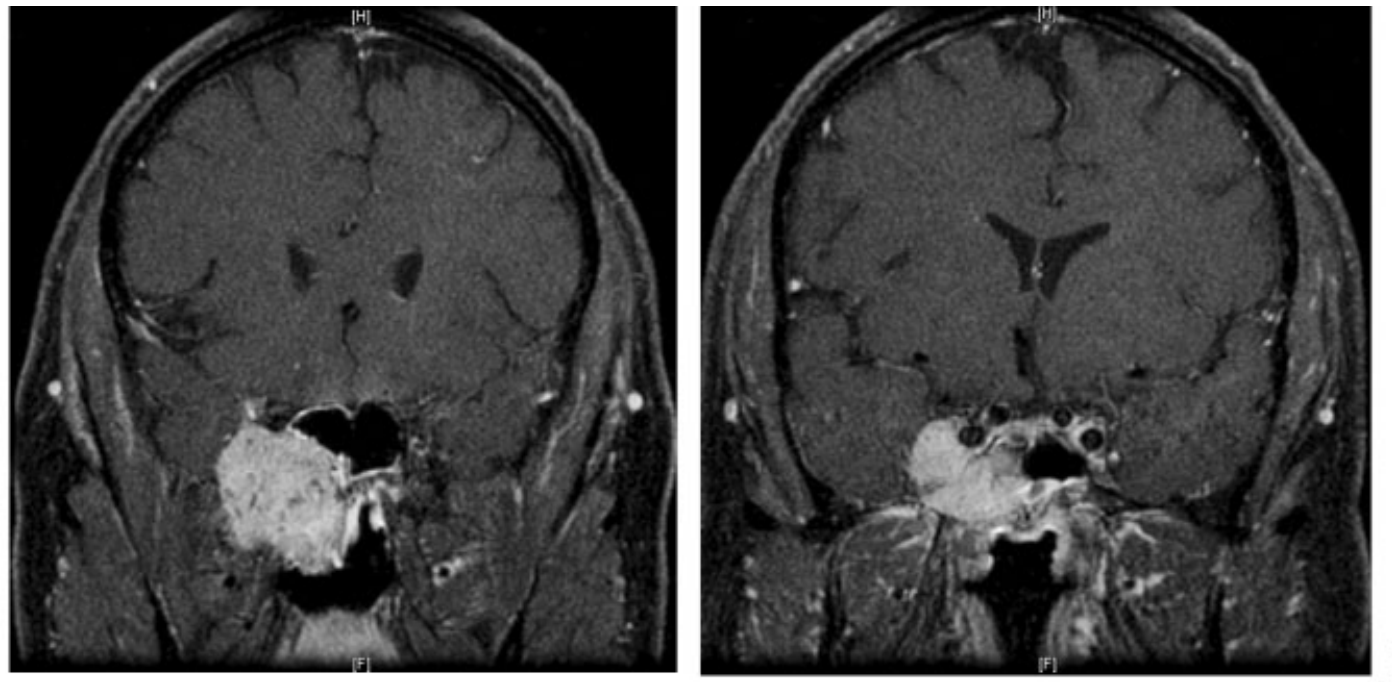

B
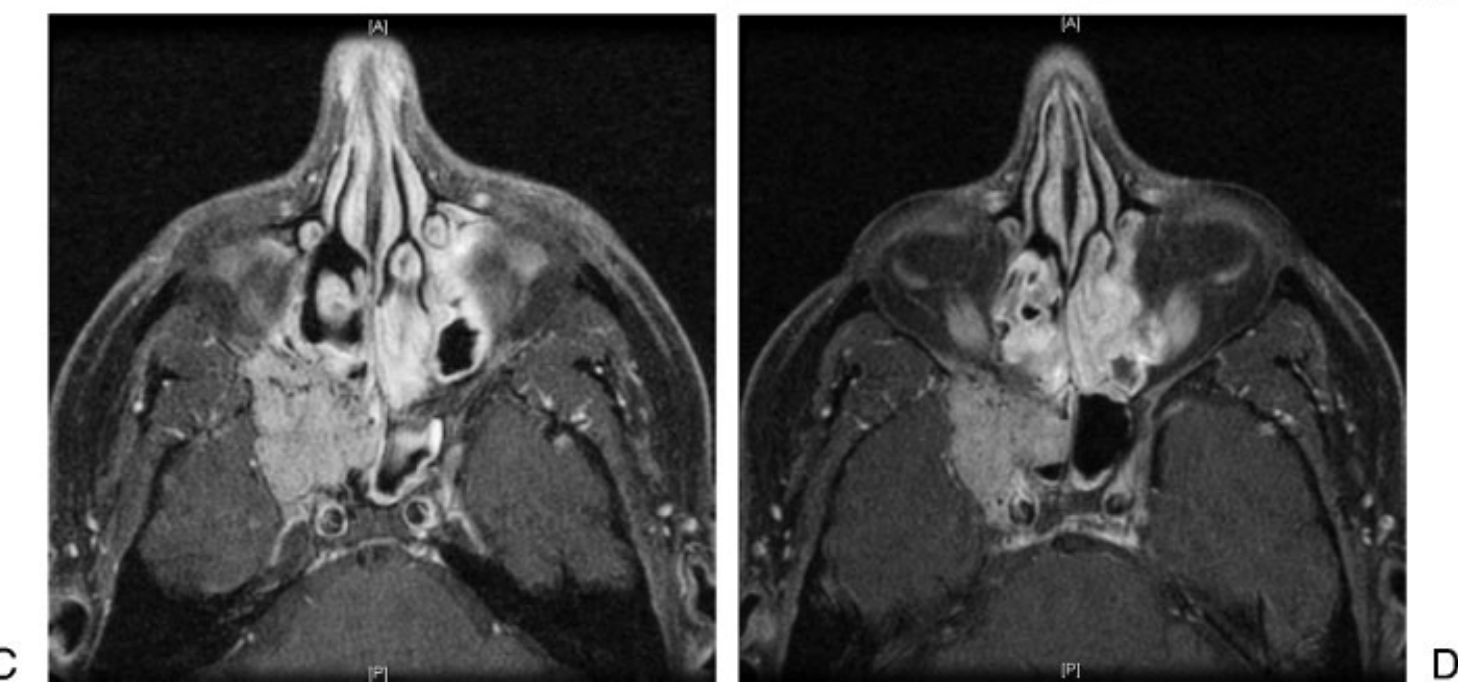

Figure 1 Magnetic resonance imaging of orbit with gadolinium enhancement (A, B) coronal sections demonstrating a lobular, solidly enhancing mass arising from the right skull base involving the right sphenopalatine foramen extending into the right nasal passage, masticator space, and parapharyngeal space. Associated osseous remodeling of the skull base can be seen. (C, D) Axial sections showing mass extending into right orbital apex, cavernous sinus with the cavernous segment of the right internal carotid artery partially encased. The mass obliterates the right foramen rotundum and vidian canal.

\section{Case Report}

The patient is a 50-year-old man with a history of a left adrenal pheochromocytoma and para-aortic paraganglioma which was surgically resected 21 years before his presentation. He did not undergo any prior genetic testing. Initial presentation began with recent episodic hypertension which was referred to a nephrologist based on his history. He had a 24-hour urine metanephrine study which showed normal urine metanephrine level, but threefold increase in plasma chromogranin A levels and an eightfold increase in plasma normetanephrine levels. He was started on phenoxybenzamine, an $\alpha$ adrenergic receptor antagonist, and metyrosine, a tyrosine hydroxylase inhibitor, for management of his hypertension from presumed recurrent abdominal paraganglioma. The patient underwent a metaiodobenzylguanidine (MIBG) scan which showed a single large focus of uptake at the right skull base corresponding to the known mass.
At this point, he was referred to our service for an otorhinolaryngologic evaluation. On a review of systems, the patient noted occasional right-sided epistaxis and nasal congestion. He did not have any cranial neuropathies. Nasal endoscopy showed a right-sided mass with mucosal hypervascularity extending from the region of the sphenopalatine foramen. The patient underwent a brain magnetic resonance imaging (MRI) scan demonstrating a $3.0 \times 4.1 \times 3.9 \mathrm{~cm}$ right sinonasal skull base mass with intracranial extension partially encasing the internal carotid artery ( Fig. 1). Subsequent computed tomography (CT) examination revealed bony remodeling of the maxillary sinus and widening of the pterygomaxillary fissure as well as bony destruction of the middle cranial fossa, lateral wall of the sphenoid sinus, and pterygoid bone (-Fig. 2). Histopathologically, biopsy of this mass demonstrated nests of cells with small, smooth contoured nuclei, and granular chromatin. Immunohistochemical stains were 

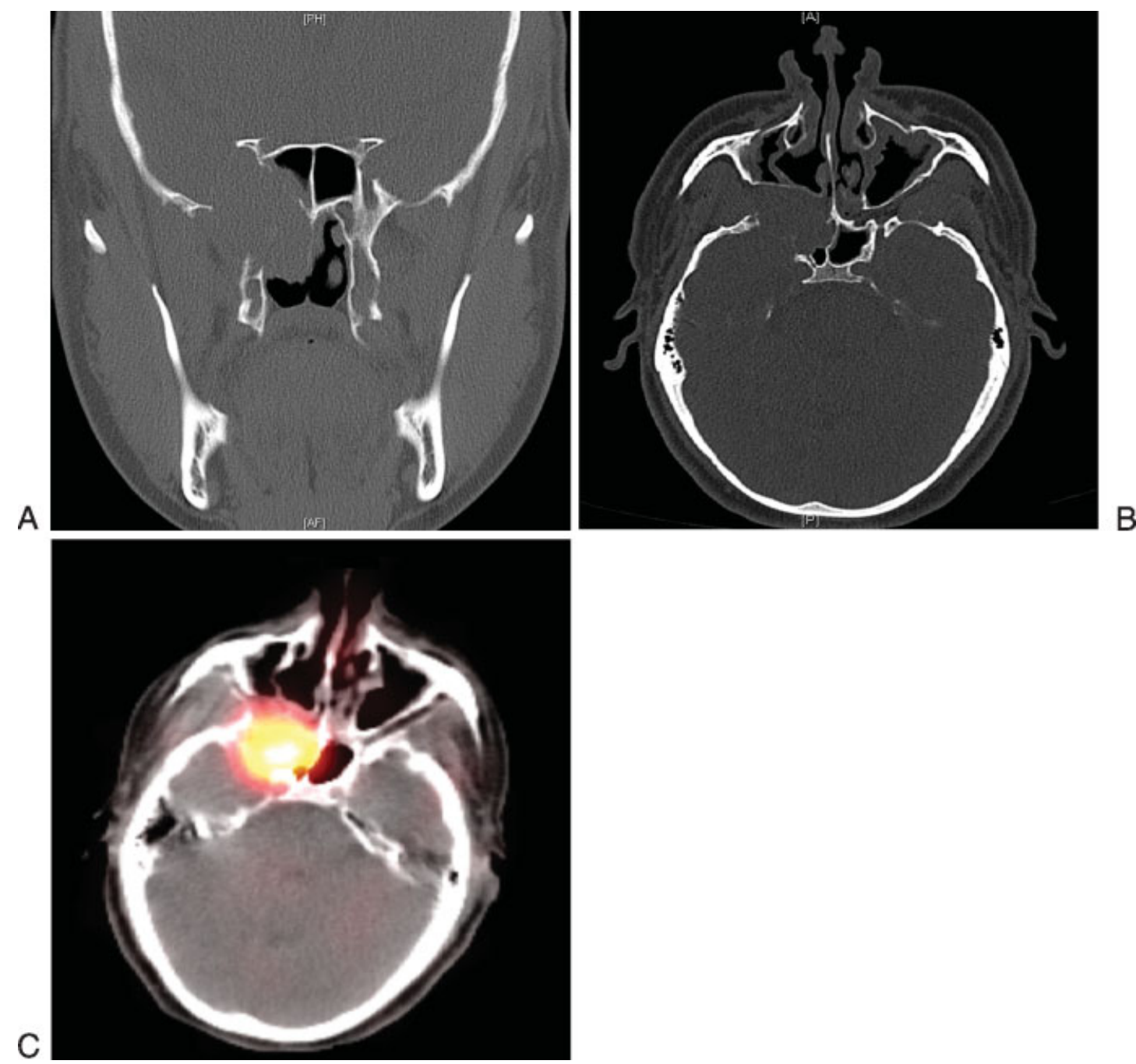

\section{B}

c

Figure 2 Computed tomography (CT) of the sinuses (A) coronal and (B) axial sections of the right sinonasal mass centered at the skull base with bony destruction of the lateral sphenoid wall as well as middle fossa. (C) Metaiodobenzylguanidine-fused CT scan showing large focus of uptake corresponding to the skull base mass.

positive for chromogranin, synaptophysin, and S-100, most consistent with a paraganglioma.

The patient subsequently underwent an endoscopic skull base resection of the paraganglioma with preoperative embolization of the internal maxillary artery (-Fig. $\mathbf{3}$ ). The patient was continued on his phenoxybenzamine and metyrosine for control of labile hypertension intra- and perioperatively. The tumor was dissected off the skull base, infratemporal fossa, and pterygoid musculature. It was traced intracranially where it was freely dissected from the dura. However, it was found to be adherent to the internal carotid artery in the region of the cavernous sinus. The decision was made to observe the cavernous carotid artery segment of tumor as a complete resection was not possible. The patient's postoperative course was unremarkable. Following the procedure, he was treated with therapeutic ${ }^{131}$ I-MIBG and external beam radiation therapy (5940 cGy). His plasma normetanephrine levels have decreased from 1190 to 394 $\mathrm{pg} / \mathrm{mL}$ (normal range $<148 \mathrm{pg} / \mathrm{mL}$ ) and chromogranin A levels decreased from 58.0 to $28.0 \mathrm{ng} / \mathrm{mL}$ (normal range 1.9 to $15.0 \mathrm{ng} / \mathrm{mL}$ ). Additionally, the patient was referred to a medical geneticist given his recurrent extra-adrenal paraganglioma. Genetic testing revealed a mutation in the SDHB gene.

\section{Discussion}

Sinonasal paragangliomas are extremely rare tumors that can arise along the skull base from the nasal cavity or the paranasal sinuses. Nasal paragangliomas originate from the middle turbinate, lateral nasal wall, or superior nasal vault. Paranasal sinus paragangliomas most often arise from the ethmoid cells and rarely from the sphenoid sinus. The origin of these tumors is believed to involve the pterygoid ganglion, sphenopalatine ganglion, and pterygoid fossa.

Only 1 to $3 \%$ of head and neck paragangliomas are functional catecholamine-secreting tumors. To date, there have been only three reported cases of secretory sinonasal paragangliomas, and no cases of a secreting skull base sinonasal paraganglioma in a patient with prior paragangliomas. Although uncommon, patients with secretory paragangliomas may present with labile hypertension which requires a 


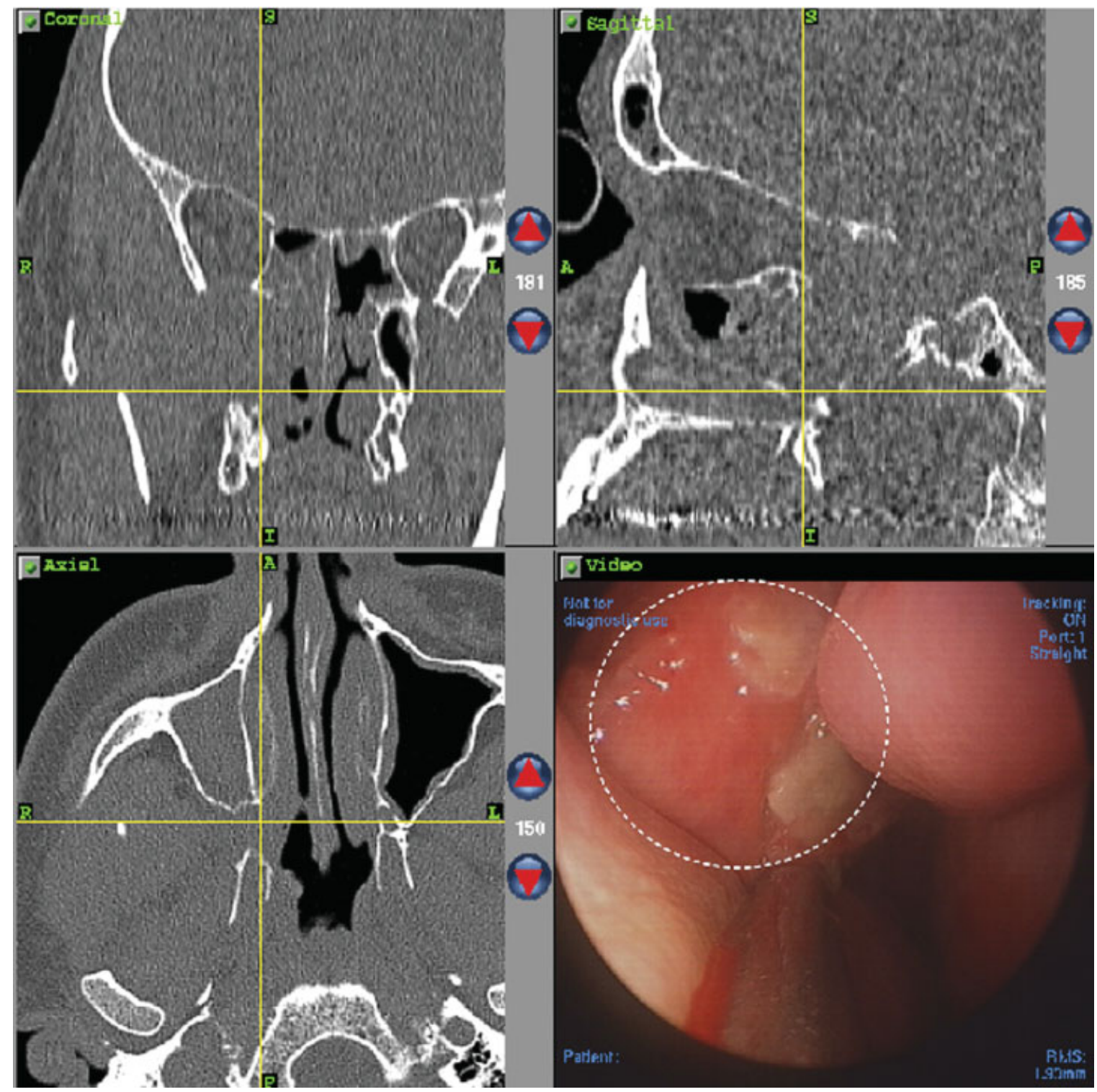

Figure 3 (A) Triplanar computed tomography (CT) scan with endoscopic intraoperative image in lower right side. The tumor (circle) extends into the right nasal cavity from the sphenopalatine region and is submucosal in nature. (B) Postresection triplanar CT scan image. Endoscopic resection extended posteriorly to the petrous segment of the internal carotid artery and laterally into the pterygopalatine fossa.

complete workup including serum and 24-hour urine catecholamine and metanephrine. In these patients, surgical intervention may be catastrophic without perioperative $\alpha$ adrenergic blockade. Additionally, evaluation for synchronous lesions using CT and/or MRI scan as well as MIBG scan is important, especially in patients with history of SDHB mutations. Patients with SDHB mutations tend to develop extra-adrenal paragangliomas with increased risk for developing tumor in the head and neck region as well as malignant and metastatic disease. SDHB has also been associated with hereditary paraganglioma and pheochromocytoma syndrome and familial genetic testing is recommended. ${ }^{10}$

Surgical resection, open or endoscopic, is the treatment of choice for paraganglioma. Complete resection of the tumor is recommended due to the potential for locally aggressive and destructive nature of sinonasal paragangliomas as well as potential for metastasis. As in this case, skull base tumors may be difficult to completely resect with clear margins due to intracranial extension or involvement of critical neurovascular structures. In these cases, postoperative high-precision radiotherapy and radionuclide ${ }^{131}$ I-MIBG can be used as adjuvant therapy. ${ }^{1}$ Although radiation therapy alone has been shown to achieve local control and symptomatic improvement, it has not been shown to achieve complete remission. ${ }^{11}$ In patients with MIBG-avid disease, radionuclide ${ }^{131}$ I-MIBG therapy can provide with symptomatic relief and tumor control through interactions with active uptake-1 mechanism at the cell membrane and neurosecretory storage granules in the cytoplasm of neuroendocrine cells. ${ }^{12}$ Phase II clinical trials have shown that ${ }^{131}$ I-MIBG therapy was effective in producing a sustained complete or partial remission in patients who have good MIBG uptake on diagnostic 


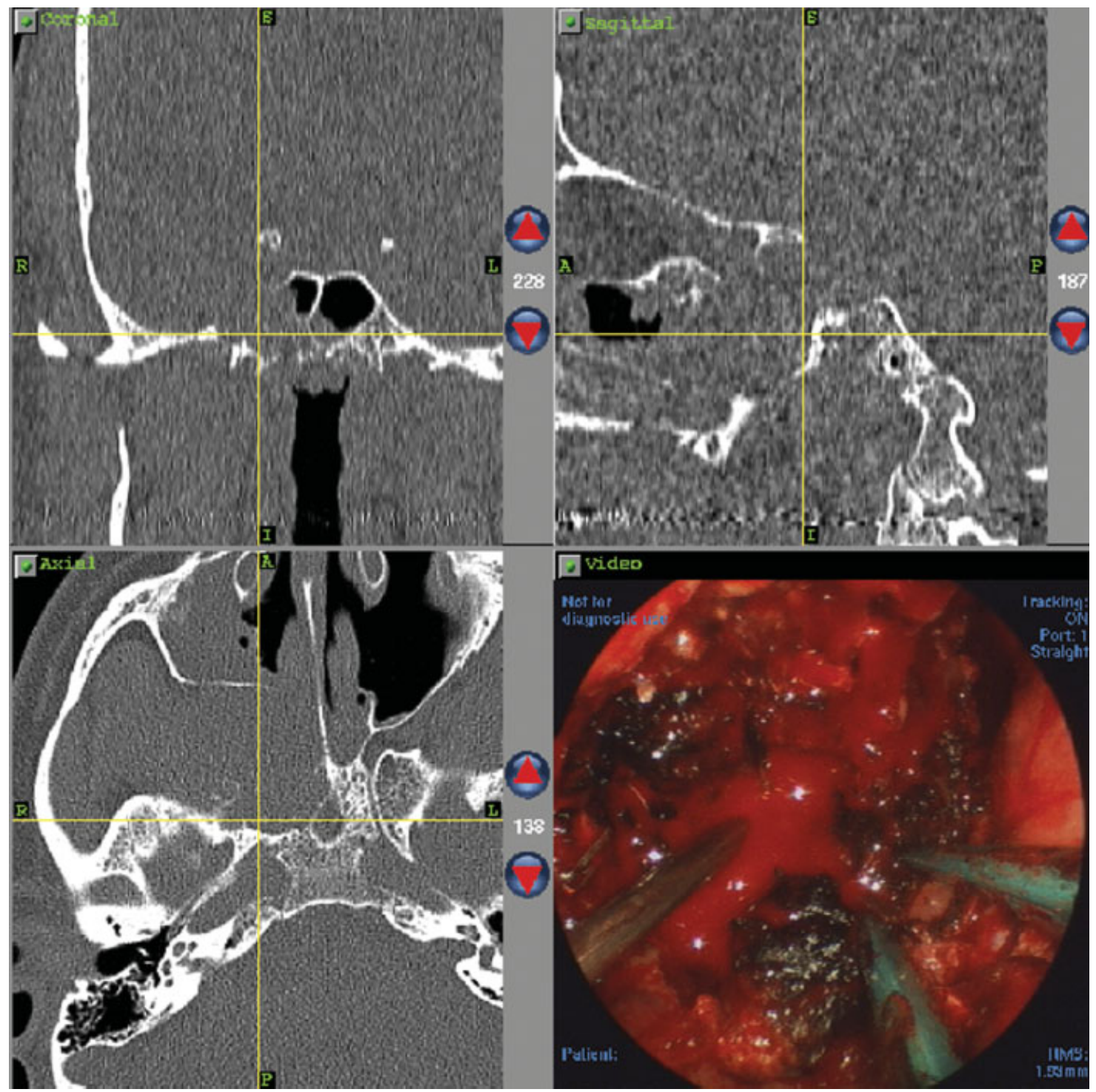

Figure 3 (Continued)

scanning. ${ }^{13}$ This patient underwent both postoperative intensity-modulated radiation therapy and therapeutic ${ }^{131}$ I-MIBG postoperatively due to tumor location and MIBG avidity.

Long-term follow-up is essential following treatment of head and neck paragangliomas. Interval biochemical assessment of urine and plasma catecholamine and metanephrine levels as well as follow-up imaging including MRI and MIBG scans can be used to assess for local and distant tumor control. This is especially important for patients with genetic mutations such as SDHB mutation who have higher probability of metastatic and metachronous tumors.

\section{Conclusion}

This case is an unusual presentation of a secreting skull base paraganglioma presenting with labile hypertension in a patient with history of prior paragangliomas and undiagnosed SDHB mutation. Complete workup for these lesions in patients with SDHB mutations should include biochemical evaluation of catecholamine and metanephrine as well as CT, MRI, and MIBG imaging of head and neck as well as thorax and abdomen for synchronous or metastatic tumor. Preoperative adrenergic blockade and angiography and embolization of the tumor are important to avoid intraoperative complications. Skull base paragangliomas may preclude complete surgical resection. In these instances, postoperative high-precision radiation and ${ }^{131} \mathrm{I}$-MIBG are viable adjuvant therapies. Long-term follow-up is essential for patients with SDHB mutations as they may develop recurrent paragangliomas.

\section{References}

1 Lee JH, Barich F, Karnell LH, et al; American College of Surgeons Commission on Cancer; American Cancer Society. National Cancer Data Base report on malignant paragangliomas of the head and neck. Cancer 2002;94(3):730-737 
2 Manolidis S, Shohet JA, Jackson CG, Glasscock ME III. Malignant glomus tumors. Laryngoscope 1999;109(1):30-34

3 Myssiorek D. Head and neck paragangliomas: an overview. Otolaryngol Clin North Am 2001;34(5):829-836, v

4 Brouwers FM, Eisenhofer G, Tao JJ, et al. High frequency of SDHB germline mutations in patients with malignant catecholamineproducing paragangliomas: implications for genetic testing. J Clin Endocrinol Metab 2006;91(11):4505-4509

5 Morales H, Castillo M, Jewells V. Paraganglioma of the sphenoid sinus: case report and review of literature. Clin Imaging 2007;31 (1):32-36

6 Kuhn JA, Aronoff BL. Nasal and nasopharyngeal paraganglioma. J Surg Oncol 1989;40(1):38-45

7 Kuhweide R, Lanser MJ, Fisch U. Catecholamine-secreting paragangliomas at the skull base. Skull Base Surg 1996;6(1):35-45

8 Apple D, Kreines K. Cushing's syndrome due to ectopic ACTH production by a nasal paraganglioma. Am J Med Sci 1982;283(1):32-35
9 Koegel L Jr, Levine HL, Waldman SR. Paraganglioma of the sphenoid sinus appearing as labile hypertension. Otolaryngol Head Neck Surg 1982;90(6):704-707

10 Amar L, Bertherat J, Baudin E, et al. Genetic testing in pheochromocytoma or functional paraganglioma. J Clin Oncol 2005;23 (34):8812-8818

11 Myssiorek D, Halaas Y, Silver C. Laryngeal and sinonasal paragangliomas. Otolaryngol Clin North Am 2001;34(5): 971-982, vii

12 Gedik GK, Hoefnagel CA, Bais E, Olmos RA. 131I-MIBG therapy in metastatic phaeochromocytoma and paraganglioma. Eur J Nucl Med Mol Imaging 2008;35(4):725-733

13 Fitzgerald PA, Goldsby RE, Huberty JP, et al. Malignant pheochromocytomas and paragangliomas: a phase II study of therapy with high-dose 131I-metaiodobenzylguanidine (131I-MIBG). Ann N Y Acad Sci 2006;1073;465-490 